\title{
Mitochondrial encephalomyopathy with lactic acidosis and stroke-like episodes in an older adult mimicking cerebral infarction: a Chinese case report
}

This article was published in the following Dove Press journal:

Clinical Interventions in Aging

\section{Gao-Li Fang' \\ Yang Zheng ${ }^{2}$ \\ Yin-Xi Zhang ${ }^{2}$}

'Department of Neurology, Hangzhou Red Cross Hospital, Hangzhou, China; ${ }^{2}$ Department of Neurology, Second Affiliated Hospital, School of Medicine, Zhejiang University, Hangzhou, China
Correspondence: Yin-Xi Zhang Department of Neurology, Second Affiliated Hospital, School of Medicine, Zhejiang University, 88 Jiefang Road, Hangzhou 310009, China

Tel $+8657 \mid 87784750$

Fax $+8657 \mid 87784750$

Email zyx-neurology@zju.edu.cn

\begin{abstract}
Few cases of mitochondrial encephalomyopathy with lactic acidosis and stroke-like episodes (MELAS) with an onset older than 60 years have been reported. Herein, we report a 63-year-old Chinese female initially suspected of ischemic infarction but was ultimately diagnosed with MELAS. Therefore, even in the elderly, a diagnosis of MELAS should be considered when encountering patients with recurrent stroke-like episodes, cognitive dysfunction, and psychotic symptoms. In order to achieve the correct diagnosis and launch the appropriate management in time, a detailed medical history together with appropriate diagnostic laboratory investigations should therefore be collected.
\end{abstract}

Keywords: mitochondrial encephalomyopathy with lactic acidosis and stroke-like episodes, adult late onset, cerebral infarction

\section{Introduction}

Mitochondrial encephalomyopathy with lactic acidosis and stroke-like episodes (MELAS) is the most common neurological mitochondrial disease. It is a clinical syndrome involving multiple organs, characterized by a myriad of symptoms such as stroke-like episodes, dementia, epilepsy, psychiatric symptoms, elevated lactic acid in the blood, myopathy, periodic headache, hearing loss, short stature, and diabetes mellitus. ${ }^{1}$ Most of them occur in adolescence, with a minority in the elderly (older than 60 years). We report a case of a 63 -year-old Chinese female who was initially suspected of ischemic infarction but was ultimately diagnosed with MELAS.

\section{Case report}

A 63-year-old woman with short stature $(148 \mathrm{~cm})$ was admitted because of a sudden onset confusion. One week ago, the patient started developing auditory and visual hallucinations, as well as disorganized speech. She was unable to communicate at that time. However, symptoms improved without treatment 1 day later. Her past medical history was notable for well-controlled hypertension and diabetes mellitus. She had a history of "cerebral infarction" 2 years ago and developed stroke-related psychotic symptoms including agitation and confusion. With the diagnosis of stroke at that time, she was treated then and improved afterward. For family history, her older sister died from "stroke" at the age of 45 . Her mother also died at an early age for unknown reasons. 

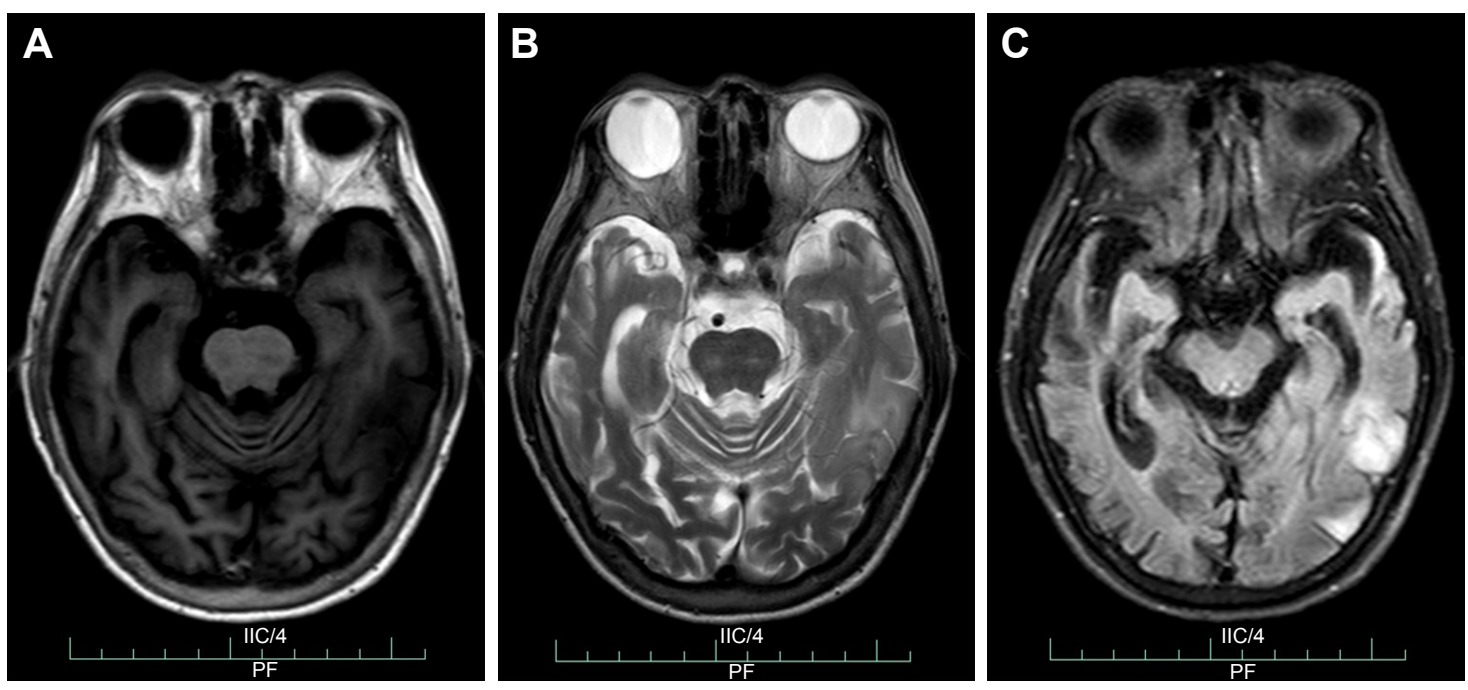

Figure I First brain MRI.

Note: Left occipital lobe lesion showed abnormal TI hypointensities (A), T2 (B), and FLAIR (C) hyperintensities. Abbreviation: FLAIR, flow attenuated inversion recovery.

The neurological examination was unremarkable except for bilateral hearing loss and bilateral positive Babinski sign. Routine laboratory investigations revealed no abnormalities excepted for a slightly elevated creatine kinase (214 U/L, reference range: 15-195 U/L). The head computed tomography scan on admission showed a lesion with low density in the left occipital lobe. The diagnosis of ischemic infarction was given considering the patient's presentation and a history of hypertension and diabetes mellitus. Treatment with aspirin and atorvastatin was started. Brain MRI afterward, however, showed a lesion in the left temporal-occipital lobe with long T1 and T2 signals, and high signals on flow attenuated inversion recovery (FLAIR) sequence (Figure 1) as well as a slightly increased signal on diffusion weighted imaging (DWI). The brain magnetic resonance angiography was normal. Unexpectedly, the patient's symptoms suddenly deteriorated 2 weeks after admission. She developed acute psychosis and right-sided hemiparesis (muscle strength of the right limbs 1/5). Mixed nonfluent aphasia was also identified on further evaluation. The patient underwent another MRI showing lesions with abnormally high signals on FLAIR and DWI with poorly defined margins along the gyri in the left occipital-temporal lobe, which was slightly enhanced after gadolinium enhancement (Figure 2). Similar episodes of psychotic symptoms and aphasia relapsed five times ever since, each lasting hours to days, and she completely recovered every time. Other tests including cerebrospinal fluid examination, autoimmune encephalitis-related antibodies, and long-term electroencephalography were nonrevealing.
On further investigation of the history, the patient recalled a progressive decline of bilateral hearing from 2 years ago and was almost deaf since last year. The lactate of venous blood was measured, showing $3.7 \mathrm{mmol} / \mathrm{L}$ at rest (reference range: $0.5-2.2 \mathrm{mmol} / \mathrm{L}$ ), and the lactic acid after exercise was not obtained because the patient refused to undergo further investigations. The patient was suspected of mitochondrial disease due to the combination of recurrent stroke-like episodes, short stature, hearing loss, and an elevated lactic acid at rest. The blood genetic test suggested a low-frequency $(<20 \%)$ mutation of m.3243A $>$ G. After being treated with arginine, vitamin $\mathrm{E}$, and coenzyme Q10 for 1 week, the patient dramatically improved. The muscle strength recovered, and there were no residual psychotic symptoms or aphasia on discharge.

This case study was approved by the institute ethics committee of the Hangzhou Red Cross Hospital. Written informed consent was obtained from the patient to publish the case details.

\section{Discussion}

MELAS was first reported in 1984 by Pavlakis et $\mathrm{al}^{2}$. Its clinical and imaging findings vary. There is often an acute to subacute onset. The disease is mostly of maternal inheritance, and genetic testing is required for diagnosis. The most common mutation is $\mathrm{A} 3243 \mathrm{G}$ point mutation of mitochondrial deoxyribonucleic acid, which accounts for about $80 \%$ of MELAS patients. ${ }^{1}$ Recurrent stroke-like episodes can occur in almost all patients and are often the initial manifestation. Patients could be easily misdiagnosed as cerebrovascular 

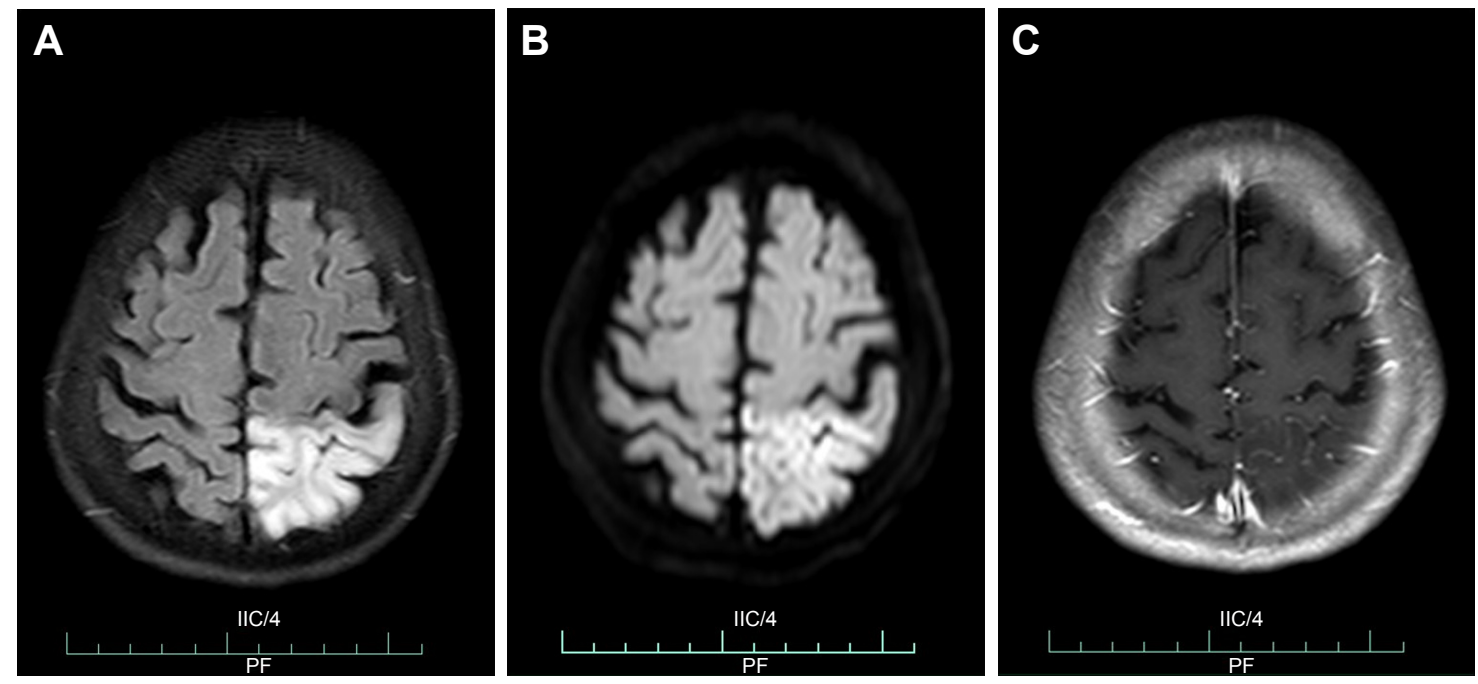

Figure 2 Subsequent brain MRI.

Note: Lesions with abnormally high signals on FLAIR (A) and DWI (B) with poorly defined margins along the gyri in the left occipital-temporal lobe, which was slightly enhanced after gadolinium enhancement (C).

Abbreviations: DWI, diffusion weigh imaging; FLAIR, flow attenuated inversion recovery.

diseases at first. Likewise, the patient in our case was previously diagnosed as "ischemic infarction" but responded poorly to treatment. Also, her mother and sister died at an early age, suggesting the possibility of maternal inheritance. Therefore, considering the presentation of our patient, her sister and mother could also be misdiagnosed. Seizures are another feature of MELAS, occurring up to $70 \%$ of MELAS patients older than 50 years. ${ }^{3}$ Patients with MELAS can also present with psychotic features, such as hallucinations and confusion. Moreover, a few patients have no obvious central nervous system symptoms but are mainly characterized by other organs/systems manifestations, for example, cardiac involvement (hypertrophic or dilated cardiomyopathy, preexcitation syndromes or conduction delay). ${ }^{4}$ The brain MRI can show a wide range of abnormalities not compatible with the distribution of blood supply. New and old lesions can be present concurrently on imaging. Temporoparietal lobe and occipital lobe are most likely affected, with no mass effect in most cases. On DWI sequence lesions with high signals can be observed along with swollen gyri. Our patient did not develop seizures as in most MELAS patients. Instead, he presented with an acute psychosis. However, the mitochondrial disease was suspected due to the combination of recurrent stroke-like episodes, fluctuations of symptoms, newly presented lesions on MRI indicating cortical laminar necrosis as well as an elevated venous lactic acid level at rest. The diagnosis of MELAS was confirmed through genetic testing afterward.

MELAS mostly presented between the age of 2 and 31 years, but rarely after the age of 40.5 Few cases with an onset older than 60 years have been reported, with only three described in detail, and the age at diagnosis was 60, 66, and 70 years, respectively. ${ }^{3,6,7}$ Hearing loss may appear long before stroke-like episodes and could be easily neglected by patients during history taking, especially for the elderly. As in this case, a careful investigation of medical history should be emphasized. Besides, studies have shown that the frequency of mitochondrial DNA mutations is related to the age of onset, indicating that patients with a low mutation frequency may present relatively at an older age. ${ }^{8}$ Therefore, the low-frequency mutation in our patient might explain her late onset of disease.

\section{Conclusion}

The present case is of the oldest MELAS patient reported in China, who was initially misdiagnosed as ischemic infarction and failed to receive a prompt treatment. In clinical practice, the diagnosis of MELAS should be considered when encountering elderly patients with recurrent stroke-like episodes, psychotic symptoms, and cognitive dysfunction. To prevent delays in treatment, a detailed medical history should therefore be collected before starting with the appropriate laboratory investigations to reach a correct diagnosis.

\section{Disclosure}

The authors report no conflicts of interest in this work.

\section{References}

1. El-Hattab AW, Adesina AM, Jones J, Scaglia F. MELAS syndrome: Clinical manifestations, pathogenesis, and treatment options. Mol Genet Metab. 2015;116(1-2):4-12. 
2. Pavlakis SG, Phillips PC, DiMauro S, De Vivo DC, Rowland LP. Mitochondrial myopathy, encephalopathy, lactic acidosis, and strokelike episodes: a distinctive clinical syndrome. Ann Neurol. 1984;16(4): 481-488.

3. Aurangzeb S, Vale T, Tofaris G, Poulton J, Turner MR. Mitochondrial encephalomyopathy with lactic acidosis and stroke-like episodes (MELAS) in the older adult. Pract Neurol. 2014;14(6):432-436.

4. Niedermayr K, Pölzl G, Scholl-Bürgi S, et al. Mitochondrial DNA mutation "m.3243A $>$ G" - heterogeneous clinical picture for cardiologists ("m.3243A>G": a phenotypic chameleon). Congenit Heart Dis. Epub 2018 Aug 21.

5. Yatsuga S, Povalko N, Nishioka J, et al. MELAS: a nationwide prospective cohort study of 96 patients in Japan. Biochim Biophys Acta. 2012; 1820(5):619-624.
6. Jones DL, Greenaway TM. Beware the thin, deaf "type 2" diabetic: maternally inherited diabetes and deafness with systemic (mitochondrial) manifestations. Intern Med J. 2004;34(8):517-518.

7. Shanske S, Coku J, Lu J, et al. The G13513A mutation in the ND5 gene of mitochondrial DNA as a common cause of MELAS or Leigh syndrome: evidence from 12 cases. Arch Neurol. 2008;65(3):368-372.

8. Qi Y, Zhang Y, Wang Z, et al. Screening of common mitochondrial mutations in Chinese patients with mitochondrial encephalomyopathies. Mitochondrion. 2007;7(1-2):147-150.
Clinical Interventions in Aging

\section{Publish your work in this journal}

Clinical Interventions in Aging is an international, peer-reviewed journal focusing on evidence-based reports on the value or lack thereof of treatments intended to prevent or delay the onset of maladaptive correlates of aging in human beings. This journal is indexed on PubMed Central, MedLine,

\section{Dovepress}

CAS, Scopus and the Elsevier Bibliographic databases. The manuscript management system is completely online and includes a very quick and fair peer-review system, which is all easy to use. Visit http://www.dovepress. com/testimonials.php to read real quotes from published authors. 